\title{
LG-Holling type II diseased predator ecosystem with Lévy noise and white noise
}

\section{Shuang Li*}

\section{"Correspondence:}

oklishuang@163.com

College of Mathematics and

Information Science, Henan Normal

University, Xinxiang, P.R. China

\section{Springer}

\begin{abstract}
This paper considers an LG-Holling type II diseased predator ecosystem with Lévy noise and white noise. In this prey-predator system, assuming that the predator population is infected by disease and divided into two classes: susceptible predator and infected predator, we show that the system has a unique global positive solution. We investigate the persistence in the mean and extinction for the population, obtain threshold conditions of extinction, persistence in the mean by Itô's formula and a comparison theorem for the stochastic system. In addition, we discuss the uniform boundedness of $p$ th moment with $p>0$ and reveal the stochastically ultimate boundedness of the system. Finally, some numerical simulations are introduced to illustrate our analytical findings.
\end{abstract}

Keywords: Leslie-Gower; Prey-predator system; Lévy noise; Persistence in the mean; Ultimate boundedness

\section{Introduction}

In ecology, prey-predator systems are always the important part, and the most classic model is Lotka-Volterra model. Out of the ordinary, Leslie introduced the following model on the premise that the carrying capacity of predator was proportional to the amount of prey [1,2]:

$$
\left\{\begin{array}{l}
\frac{d N_{1}}{d t}=\left(r_{1}-a_{1} N_{1}-b_{2} N_{2}\right) N_{1}, \\
\frac{d N_{2}}{d t}=\left(r_{2}-b_{2} \frac{N_{2}}{N_{1}}\right) N_{2},
\end{array}\right.
$$

here $N_{1}$ and $N_{2}$ represented the densities of prey and predator at time $t$, respectively. $r_{1}$ and $r_{2}$ were the intrinsic growth rates for prey and predator populations. $b_{2}$ was conversion rate. Leslie assumed that the predator population still grew logistically with carrying capacity $\frac{r_{2} N_{1}}{b_{2}}$.

Leslie assumed that the growth of predator population still satisfied the logistic rule, but the carrying capacity, which was distinguished from the constant in the common model, was determined by the density of prey population. Recently, there have been many experts and researchers studying or improving a Leslie type prey-predator model. Time delay, impulse, food chain, disease, environment pollution, reaction diffusion and so on, have been introduced into the model, see [3-8].

(c) The Author(s) 2018. This article is distributed under the terms of the Creative Commons Attribution 4.0 International License (http://creativecommons.org/licenses/by/4.0/), which permits unrestricted use, distribution, and reproduction in any medium, provided you give appropriate credit to the original author(s) and the source, provide a link to the Creative Commons license, and indicate if changes were made. 
In [9], Sarwardi introduced a contagious disease among the predators in a predator-prey ecosystem, modeled via the following equations with nonnegative parameters:

$$
\left\{\begin{array}{l}
\frac{d x}{d t}=a_{1} x-b_{1} x^{2}-\frac{c_{1} x y}{x+k_{1}}-\frac{p c_{1} x z}{x+k_{1}} \\
\frac{d y}{d t}=a_{2} y-\frac{c_{2} y(y+z)}{x+k_{2}}-\lambda y z \\
\frac{d z}{d t}=\lambda y z+a_{3} z-\frac{c_{3} z(y+z)}{x+k_{2}}
\end{array}\right.
$$

The system incorporates a Holling type II functional response and a modified version of the Leslie-Gower one. In system (2), the following assumptions are made:

(A1) $x(t)$ represents the density of prey population at time $t$, and it is assumed that the disease spreads only among the predator, $y(t)$ and $z(t)$ denote the densities of susceptible predator and infected predator at time $t$, the total predator population is $n(t)=y(t)+z(t)$. The disease transmission rate is $\lambda$.

(A2) In the absence of predators, the prey population grows logistically with intrinsic growth rate $a_{1}>0$ and carrying capacity $a_{1} b_{1}^{-1} . c_{1}$ and $p c_{1}$ are the predation rates for susceptible and infected predator. Because of disease infection, the predation ability for the infected predator is a bit less than that for the susceptible predator; therefore, $0<p<1$.

(A3) Here $a_{2}, a_{3}$ are the per capita growth rates of each predator population. The parameters $k_{1}, k_{2}$ respectively represent the half saturation constants of prey and predator populations. In addition, the authors introduce intraspecific competition among the predators' sound and infected subpopulations with parameters $c_{2}$ and $c_{3}$, for which $c_{2}>c_{3}$.

The authors studied the stability of equilibrium point of system (2) and persistence.

Population growth in the natural world is inevitably affected by the environmental perturbations. In the past decades, white noise was introduced into the model to simulate the stochastic perturbation of environment. However, sudden environmental perturbation, such as earthquakes, hurricanes, the flood, etc., cannot be modeled by white noise, which is often used to describe those stable and continuous stochastic interferences. Bao et al. in $[10,11]$ firstly proposed that these phenomena could be described by Lévy noise. At present, there are many research papers considering Lévy noise [6,12-17]. In this paper, we use white noise and Lévy noise to simulate the random change of environment. The following system with jumps is considered:

$$
\left\{\begin{aligned}
d x(t)= & {\left[a_{1} x(t)-b_{1} x^{2}(t)-\frac{c_{1} x(t) y(t)}{x(t)+k_{1}}-\frac{\tilde{p} c_{1} x(t) z(t)}{x(t)+k_{1}}\right] d t } \\
& +\sigma_{1} x(t) d B_{1}(t)+\int_{\mathbb{Y}} \gamma_{1}(u) x\left(t^{-}\right) \tilde{N}(d t, d u), \\
d y(t)= & {\left[a_{2} y(t)-\frac{c_{2} y(t)(y(t)+z(t))}{x(t)+k_{2}}-\beta y(t) z(t)\right] d t } \\
& +\sigma_{2} y(t) d B_{2}(t)+\int_{\mathbb{Y}} \gamma_{2}(u) y\left(t^{-}\right) \tilde{N}(d t, d u), \\
d z(t)= & {\left[\beta y(t) z(t)+a_{3} z(t)-\frac{c_{3} z(t)(y(t)+z(t))}{x(t)+k_{2}}\right] d t } \\
& +\sigma_{3} z(t) d B_{3}(t)+\int_{\mathbb{Y}} \gamma_{3}(u) z\left(t^{-}\right) \tilde{N}(d t, d u),
\end{aligned}\right.
$$

with the initial value $\left(x_{0}, y_{0}, z_{0}\right) \in R_{+}^{3}$. For convenience, we use $\tilde{p}$ and $\beta$ instead of $p$ and $\lambda$ in system (3), respectively. $B_{1}(t), B_{2}(t)$ and $B_{3}(t)$ are mutually independent Brownian motions defined on a complete probability space $(\Omega, \mathcal{F}, \mathcal{P})$ with a filtration $\left\{\mathcal{F}_{t}\right\}_{t \in R_{+}}$satisfying the usual conditions. $\sigma_{i}^{2}(i=1,2,3)$ denotes the intensity of white noise. In system (3), $x\left(t^{-}\right), y\left(t^{-}\right)$and $z\left(t^{-}\right)$are the left limits of $x(t), y(t)$ and $z(t)$, respectively. $N$ is a Poisson 
counting measure with characteristic measure $\lambda$ on a measurable subset $\mathbb{Y}$ of $(0,+\infty)$ with $\lambda(\mathbb{Y})<\infty, \tilde{N}(d t, d u)=N(d t, d u)-\lambda(d u) d t, \gamma_{i}: \mathbb{Y} \times \Omega \rightarrow \mathbb{R}$ is bounded and continuous with respect to $\lambda$, and is $\mathcal{B}(\mathbb{Y}) \times \mathcal{F}_{t}$-measurable, $i=1,2,3$.

For simplicity, we introduce the following notations:

$$
\begin{aligned}
& w_{i}=a_{i}-\frac{\sigma_{i}^{2}}{2}-\int_{\mathbb{Y}}\left[\gamma_{i}(u)-\ln \left(1+\gamma_{i}(u)\right)\right] \lambda(d u), \quad i=1,2,3 . \\
& R_{+}^{3}=\left\{\left(\eta_{1}, \eta_{2}, \eta_{3}\right)^{T} \in R^{3} \mid \eta_{i}>0, i=1,2,3\right\} .
\end{aligned}
$$

Assumption 1 There exists a constant $c$ satisfying

$$
\int_{\mathbb{Y}}\left[\ln \left(1+\gamma_{i}(u)\right)\right]^{2} \lambda(d u)<c, \quad i=1,2,3 .
$$

Throughout this paper, we assume that Assumption 1 is true.

\section{The existence of global positive solution}

Theorem 1 For any initial value $\left(x_{0}, y_{0}, z_{0}\right) \in R_{+}^{3}$, system (3) has a unique global solution $(x(t), y(t), z(t)) \in R_{+}^{3}$ for $t \geq 0$, and the solution will remain in $R_{+}^{3}$ almost surely.

Proof At first, let us consider the following system:

$$
\left\{\begin{aligned}
d u(t)= & {\left[w_{1}-b_{1} \exp (u(t))-\frac{c_{1} \exp \left(v_{1}(t)\right)}{\exp (u(t))+k_{1}}-\frac{\tilde{p} c_{1} \exp \left(v_{2}(t)\right)}{\exp (u(t))+k_{1}}\right] d t } \\
& +\sigma_{1} d B_{1}(t)+\int_{\mathbb{Y}} \ln \left(1+\gamma_{1}(u)\right) \tilde{N}(d t, d u), \\
d v_{1}(t)= & {\left[w_{2}-\frac{c_{2}\left(\exp \left(v_{1}(t)\right)+\exp \left(v_{2}(t)\right)\right)}{\exp (u(t))+k_{2}}-\beta \exp \left(v_{2}(t)\right)\right] d t } \\
& +\sigma_{2} d B_{2}(t)+\int_{\mathbb{Y}} \ln \left(1+\gamma_{2}(u)\right) \tilde{N}(d t, d u), \\
d v_{2}(t)= & {\left[w_{3}+\beta \exp \left(v_{1}(t)\right)-\frac{c_{3}\left(\exp \left(v_{1}(t)\right)+\exp \left(v_{2}(t)\right)\right)}{\exp (u(t)))+k_{2}}\right] d t } \\
& +\sigma_{3} d B_{3}(t)+\int_{\mathbb{Y}} \ln \left(1+\gamma_{3}(u)\right) \tilde{N}(d t, d u),
\end{aligned}\right.
$$

on $t \geq 0$ with the initial data $\left(\ln x_{0}, \ln y_{0}, \ln z_{0}\right)$. Clearly, the coefficients of system (4) satisfy the local Lipschitz condition, then there is a unique local solution on $\left[0, \tau_{e}\right)$, where $\tau_{e}$ is the explosion time. Therefore, by Itô's formula, $\left(\exp (u(t)), \exp \left(v_{1}(t)\right), \exp \left(v_{2}(t)\right)\right)$ is the unique positive local solution of system (3) with the initial value $\left(x_{0}, y_{0}, z_{0}\right) \in R_{+}^{3}$. Whereafter, we will show that $\tau_{e}=\infty$, namely the solution of system (3) is globally existent.

Consider the stochastic differential equations:

$$
\left\{\begin{aligned}
d \Phi_{1}(t)= & \Phi_{1}(t)\left[a_{1}-b_{1} \Phi_{1}(t)\right] d t+\sigma_{1} \Phi_{1}(t) d B_{1}(t)+\int_{\mathbb{Y}} \gamma_{1}(u) \Phi_{1}\left(t^{-}\right) \tilde{N}(d t, d u), \\
d \Phi_{2}(t)= & \Phi_{2}(t)\left[a_{2}-\frac{c_{2} \Phi_{2}(t)}{\Phi_{1}(t)+k_{2}}\right] d t+\sigma_{2} \Phi_{2}(t) d B_{2}(t)+\int_{\mathbb{Y}} \gamma_{2}(u) \Phi_{2}\left(t^{-}\right) \tilde{N}(d t, d u), \\
d \Phi_{3}(t)= & \Phi_{3}(t)\left[\beta \Phi_{2}(t)+a_{3}-\frac{c_{3} \Phi_{3}(t)}{\Phi_{1}(t)+k_{2}}\right] d t \\
& +\sigma_{3} \Phi_{3}(t) d B_{3}(t)+\int_{\mathbb{Y}} \gamma_{3}(u) \Phi_{3}\left(t^{-}\right) \tilde{N}(d t, d u)
\end{aligned}\right.
$$

and

$$
\left\{\begin{aligned}
d \Psi_{1}(t)= & \Psi_{1}(t)\left[a_{1}-b_{1} \Psi_{1}(t)-\frac{c_{1} \Phi_{2}(t)}{k_{1}}-\frac{\tilde{p} c_{1} \Phi_{3}(t)}{k_{1}}\right] d t \\
& +\sigma_{1} \Psi_{1}(t) d B_{1}(t)+\int_{\mathbb{Y}} \gamma_{1}(u) \Psi_{1}\left(t^{-}\right) \tilde{N}(d t, d u), \\
d \Psi_{2}(t)= & \Psi_{2}(t)\left[a_{2}-\frac{c_{2} \Psi_{2}(t)}{k_{2}}-\frac{c_{2} \Phi_{3}(t)}{k_{2}}-\beta \Phi_{3}(t)\right] d t \\
& +\sigma_{2} \Psi_{2}(t) d B_{2}(t)+\int_{\mathbb{Y}} \gamma_{2}(u) \Psi_{2}\left(t^{-}\right) \tilde{N}(d t, d u), \\
d \Psi_{3}(t)= & \Psi_{3}(t)\left[\beta \Psi_{2}(t)+a_{3}-\frac{c_{3} \Phi_{2}(t)}{k_{2}}-\frac{c_{3} \Psi_{3}(t)}{k_{2}}\right] d t \\
& +\sigma_{3} \Psi_{3}(t) d B_{3}(t)+\int_{\mathbb{Y}} \gamma_{3}(u) \Psi_{3}\left(t^{-}\right) \tilde{N}(d t, d u) .
\end{aligned}\right.
$$


According to the comparison theorem for stochastic differential equations in [18], we can get that, for $t \in\left[0, \tau_{e}\right)$,

$$
\Psi_{1}(t) \leq x(t) \leq \Phi_{1}(t), \quad \Psi_{2}(t) \leq y(t) \leq \Phi_{2}(t), \quad \Psi_{3}(t) \leq z(t) \leq \Phi_{3}(t), \quad \text { a.s. }
$$

By virtue of Lemma 4.2 in [10], we can see that $\Phi_{i}(t)$ and $\Psi_{i}(t)(i=1,2,3)$ will not explode in any finite time, which means that $\Phi_{i}(t)$ and $\Psi_{i}(t)$ are existent on $t \geq 0$. Thus, we obtain $\tau_{e}=+\infty$, the proof of Theorem 1 is completed.

According to Lemma 4.4 in [10] and equations (5), we know that

$$
\limsup _{t \rightarrow \infty} \frac{\ln \Phi_{i}(t)}{\ln t} \leq 1, \quad \text { a.s. }
$$

for $i=1,2,3$, when

$$
\sup _{t \geq 0} \int_{0}^{t} \int_{\mathbb{Y}} \exp (s-t)\left[\gamma_{i}(u)-\ln \left(1+\gamma_{i}(u)\right)\right] \lambda(d u) d s<\infty .
$$

Noting the $\operatorname{limit} \lim _{t \rightarrow \infty} \frac{\ln t}{t}=0, \lim \sup _{t \rightarrow \infty} \frac{\ln \Phi_{i}(t)}{t} \leq 0$ is obtained for $i=1,2,3$, a.s. It then follows from the comparison theorem of [18] that

$$
\begin{aligned}
& \limsup _{t \rightarrow \infty} \frac{\ln x(t)}{t} \leq \limsup _{t \rightarrow \infty} \frac{\ln \Phi_{1}(t)}{t} \leq 0, \quad \text { a.s., } \\
& \limsup _{t \rightarrow \infty} \frac{\ln y(t)}{t} \leq \limsup _{t \rightarrow \infty} \frac{\ln \Phi_{2}(t)}{t} \leq 0, \quad \text { a.s., } \\
& \limsup _{t \rightarrow \infty} \frac{\ln z(t)}{t} \leq \limsup _{t \rightarrow \infty} \frac{\ln \Phi_{3}(t)}{t} \leq 0, \quad \text { a.s. }
\end{aligned}
$$

Thus, we have a lemma as follows.

Lemma 1 Assume that, for any $t \geq 0$ and $i=1,2,3$,

$$
\sup _{t \geq 0} \int_{0}^{t} \int_{\mathbb{Y}} \exp (s-t)\left[\gamma_{i}(u)-\ln \left(1+\gamma_{i}(u)\right)\right] \lambda(d u) d s<\infty
$$

Then, for any initial value $\left(x_{0}, y_{0}, z_{0}\right) \in R_{+}^{3}$, the solution $(x(t), y(t), z(t))$ of system (3) satisfies

$$
\limsup _{t \rightarrow \infty} \frac{\ln x(t)}{t} \leq 0, \quad \limsup _{t \rightarrow \infty} \frac{\ln y(t)}{t} \leq 0, \quad \limsup _{t \rightarrow \infty} \frac{\ln z(t)}{t} \leq 0, \quad \text { a.s. }
$$

\section{Persistence in the mean and extinction}

Lemma 2 (see Liu et al. [14]) Suppose that $Z(t) \in C\left(\Omega \times[0, \infty), R_{+}\right)$, and let $\int_{\mathbb{Y}}\left[\ln \left(1+\gamma_{i}(u)\right)\right]^{2} \lambda(d u)<c, i=1,2$, hold.

(I) If there exist two positive constants $T$ and $\delta_{0}$ such that

$$
\ln Z(t) \leq \delta t-\delta_{0} \int_{0}^{t} Z(s) d s+\alpha B(t)+\sum_{i=1}^{2} \delta_{i} \int_{0}^{t} \int_{\mathbb{Y}} \ln \left(1+\gamma_{i}(u)\right) \tilde{N}(d s, d u), \quad \text { a.s. }
$$


for all $t \geq T$, where $\alpha, \delta_{1}$ and $\delta_{2}$ are constants, then

$$
\begin{cases}\limsup _{t \rightarrow \infty} \frac{1}{t} \int_{0}^{t} Z(s) d s \leq \delta / \delta_{0}, & \text { a.s., if } \delta \geq 0 \\ \lim _{t \rightarrow \infty} Z(t)=0, & \text { a.s., if } \delta<0 .\end{cases}
$$

(II) If there exist three positive constants $T, \delta$ and $\delta_{0}$ such that

$$
\ln Z(t) \geq \delta t-\delta_{0} \int_{0}^{t} Z(s) d s+\alpha B(t)+\sum_{i=1}^{2} \delta_{i} \int_{0}^{t} \int_{\mathbb{Y}} \ln \left(1+\gamma_{i}(u)\right) \tilde{N}(d s, d u), \quad \text { a.s. }
$$

for all $t \geq T$, then $\liminf _{t \rightarrow \infty} \frac{1}{t} \int_{0}^{t} Z(s) d s \geq \delta / \delta_{0}$, a.s.

Definition 1 Assume $m(t)$ is the density of a population at time $t$ :

(1) If $\lim _{t \rightarrow \infty} m(t)=0$, a.s., then species $m(t)$ is said to be extinct.

(2) If $\lim _{t \rightarrow \infty} \frac{1}{t} \int_{0}^{t} m(s) d s>0$, a.s., then species $m(t)$ is said to be stable in the mean.

(3) If $\liminf _{t \rightarrow \infty} \frac{1}{t} \int_{0}^{t} m(s) d s>0$, a.s., then species $m(t)$ is said to be strongly persistent in the mean.

(4) If $\lim \sup _{t \rightarrow \infty} \frac{1}{t} \int_{0}^{t} m(s) d s>0$, a.s., then species $m(t)$ is said to be weakly persistent in the mean.

Obviously, if species $m(t)$ is stable in the mean, it must be strongly or weakly persistent in the mean.

Making use of Itô's formula to system (3) gives

$$
\left\{\begin{aligned}
d \ln x(t)= & {\left[w_{1}-b_{1} x(t)-\frac{c_{1} y(t)}{x(t)+k_{1}}-\frac{\tilde{p} c_{1} z(t)}{x(t)+k_{1}}\right] d t } \\
& +\sigma_{1} d B_{1}(t)+\int_{\mathbb{Y}} \ln \left(1+\gamma_{1}(u)\right) \tilde{N}(d t, d u), \\
d \ln y(t)= & {\left[w_{2}-\frac{c_{2}(y(t)+z(t))}{x(t)+k_{2}}-\beta z(t)\right] d t+\sigma_{2} d B_{2}(t)+\int_{\mathbb{Y}} \ln \left(1+\gamma_{2}(u)\right) \tilde{N}(d t, d u), } \\
d \ln z(t)= & {\left[w_{3}+\beta y(t)-\frac{c_{3}(y(t)+z(t))}{x(t)+k_{2}}\right] d t+\sigma_{3} d B_{3}(t)+\int_{\mathbb{Y}} \ln \left(1+\gamma_{3}(u)\right) \tilde{N}(d t, d u) . }
\end{aligned}\right.
$$

For both sides of system (7), integrating from 0 to $t$ and multiplying by $\frac{1}{t}$, (7) equals

$$
\left\{\begin{array}{l}
\frac{\ln x(t)-\ln x_{0}}{t}=w_{1}-b_{1} \frac{1}{t} \int_{0}^{t} x(s) d s-\frac{1}{t} \int_{0}^{t} \frac{c_{1} y(s)}{x(s)+k_{1}} d s-\frac{1}{t} \int_{0}^{t} \frac{\tilde{p} c_{1} z(s)}{x(s)+k_{1}} d s+\frac{\sigma_{1} B_{1}(t)}{t}+\frac{M_{1}(t)}{t}, \\
\frac{\ln y(t)-\ln y_{0}}{t}=w_{2}-\frac{1}{t} \int_{0}^{t} \frac{c_{2}(y(s)+z(s))}{x(s)+k_{2}} d s-\beta \frac{1}{t} \int_{0}^{t} z(s) d s+\frac{\sigma_{2} B_{2}(t)}{t}+\frac{M_{2}(t)}{t}, \\
\frac{\ln z(t)-\ln z_{0}}{t}=w_{3}+\beta \frac{1}{t} \int_{0}^{t} y(s) d s-\frac{1}{t} \int_{0}^{t} \frac{c_{3}(y(s)+z(s))}{x(s)+k_{2}} d s+\frac{\sigma_{3} B_{3}(t)}{t}+\frac{M_{3}(t)}{t},
\end{array}\right.
$$

where $M_{i}(t)=\int_{0}^{t} \int_{\mathbb{Y}} \ln \left(1+\gamma_{i}(u)\right) \tilde{N}(d s, d u), i=1,2,3$.

$M_{i}(t)$ are local martingale, by Proposition 2.4 of [19] and Assumption 1, its quadratic variations are

$$
\begin{aligned}
\left\langle M_{i}, M_{i}\right\rangle(t) & =\int_{0}^{t} \int_{\mathbb{Y}}\left[\ln \left(1+\gamma_{i}(u)\right)\right]^{2} \lambda(d u) d s \\
& =t \int_{\mathbb{Y}}\left[\ln \left(1+\gamma_{i}(u)\right)\right]^{2} \lambda(d u) \\
& <c t, \quad i=1,2,3 .
\end{aligned}
$$

According to the law of strong large numbers, we have

$$
\lim _{t \rightarrow \infty} \frac{M_{i}(t)}{t}=0, \quad \text { a.s. } i=1,2,3 .
$$


Obviously,

$$
\lim _{t \rightarrow \infty} \frac{B_{i}(t)}{t}=0, \quad \text { a.s. } i=1,2,3 .
$$

In the following, we discuss the persistence in the mean and extinction for prey and predator populations.

\section{Theorem 2}

(i) If $w_{i}<0(i=1,2,3)$, the prey population $x(t)$ and both the predator populations $y(t)$ and $z(t)$ will be extinct with probability 1 .

(ii) If $w_{1}<0, w_{2}<0$ and $w_{3}>0$, the prey population $x(t)$ and the susceptible predator population $y(t)$ will go to extinction, a.s., the infected predator population $z(t)$ will be stable in the mean, i.e., $\lim _{t \rightarrow \infty} \frac{1}{t} \int_{0}^{t} z(s) d s=\frac{w_{3} k_{2}}{c_{3}}$.

(iii) If $w_{1}<0, w_{2}>0$ and $w_{3}<0$, the prey population $x(t)$ will go to extinction, the growth of the predator population is divided into the following two cases:

(a) when $w_{3}+\frac{\beta k_{2}}{c_{2}} w_{2}<0$, then the susceptible predator $y(t)$ will be stable in the mean, $\lim _{t \rightarrow \infty} \frac{1}{t} \int_{0}^{t} y(s) d s=\frac{w_{2} k_{2}}{c_{2}}$, and the infected predator $z(t)$ will be extinct, a.s.

(b) when $w_{3}+\frac{\beta k_{2}}{c_{2}} w_{2}>0, w_{2}>\frac{\left(c_{2}+k_{2} \beta\right)\left(w_{3} c_{2}+\beta k_{2} w_{2}\right)}{c_{2} c_{3}}$ and $w_{3}+\beta Q>\frac{c_{3} w_{2}}{c_{2}}$, where $Q=\frac{k_{2}}{c_{2}}\left[w_{2}-\frac{\left(c_{2}+k_{2} \beta\right)\left(w_{3} c_{2}+\beta k_{2} w_{2}\right)}{c_{2} c_{3}}\right]$, the whole predator population $y(t)$ and $z(t)$ will be strongly persistent in the mean, a.s.

(iv) If $w_{1}>0, w_{2}<0$ and $w_{3}<0$, then the prey population $x(t)$ will be stable in the mean, namely $\lim _{t \rightarrow \infty} \frac{1}{t} \int_{0}^{t} x(s) d s=\frac{w_{1}}{b_{1}}$, a.s. Both the susceptible predator $y(t)$ and the infected predator $z(t)$ will die out, a.s.

(v) Assume that, for any $t \geq 0$ and $i=1,2,3$,

$$
\sup _{t \geq 0} \int_{0}^{t} \int_{\mathbb{Y}} \exp (s-t)\left[\gamma_{i}(u)-\ln \left(1+\gamma_{i}(u)\right)\right] \lambda(d u) d s<\infty,
$$

then if $w_{1}>\frac{\left(w_{2}+w_{3}\right) k_{2} c_{1}}{\left(c_{2}+c_{3}+k_{2} \beta\right) k_{1}}, w_{2}>0$ and $w_{3}>0$, then all the populations will be strongly persistent in the mean, where $\liminf _{t \rightarrow \infty}\left[\frac{1}{t} \int_{0}^{t} x(s) d s\right] \geq \frac{w_{1}}{b_{1}}-\frac{\left(w_{2}+w_{3}\right) k_{2} c_{1}}{b_{1} k_{1}\left(c_{2}+c_{3}+k_{2} \beta\right)}$ and $\liminf _{t \rightarrow \infty} \frac{1}{t} \int_{0}^{t}[y(s)+z(s)] d s \geq \frac{\left(w_{2}+w_{3}\right) k_{2}}{c_{2}+c_{3}+k_{2} \beta}$, a.s.

Proof Making use of (8), we get

$$
\left\{\begin{array}{l}
\frac{1}{t} \ln \frac{x(t)}{x_{0}} \leq w_{1}-b_{1} \frac{1}{t} \int_{0}^{t} x(s) d s+\frac{\sigma_{1} B_{1}(t)}{t}+\frac{M_{1}(t)}{t} \\
\frac{1}{t} \ln \frac{y(t)}{y_{0}} \leq w_{2}-\frac{1}{t} \int_{0}^{t} \frac{c_{2} y(s)}{x(s)+k_{2}} d s+\frac{\sigma_{2} B_{2}(t)}{t}+\frac{M_{2}(t)}{t} \\
\frac{1}{t} \ln \frac{z(t)}{z_{0}} \leq w_{3}+\beta \frac{1}{t} \int_{0}^{t} y(s) d s-\frac{1}{t} \int_{0}^{t} \frac{c_{3} z(s)}{x(s)+k_{2}} d s+\frac{\sigma_{3} B_{3}(t)}{t}+\frac{M_{3}(t)}{t}
\end{array}\right.
$$

(i) According to (11),

$$
\frac{1}{t} \ln \frac{x(t)}{x_{0}} \leq w_{1}-b_{1} \frac{1}{t} \int_{0}^{t} x(s) d s+\frac{\sigma_{1} B_{1}(t)}{t}+\frac{M_{1}(t)}{t}
$$

noting (9) and (10), by virtue of Lemma 2, we can get $\lim _{t \rightarrow \infty} x(t)=0$ when $w_{1}<0$. Then, for an arbitrary small constant $\varepsilon_{1}$ which satisfies $0<\varepsilon_{1}<-\frac{w_{3}}{\beta}$, there exists a sufficiently 
large number $T_{1}\left(T_{1}>0\right)$ such that

$$
-\varepsilon_{1} \leq x(t) \leq \varepsilon_{1}
$$

for $t \geq T_{1}$; therefore,

$$
\frac{1}{t} \ln \frac{y(t)}{y_{0}} \leq w_{2}-\frac{1}{t} \int_{0}^{t} \frac{c_{2} y(s)}{\varepsilon_{1}+k_{2}} d s+\frac{\sigma_{2} B_{2}(t)}{t}+\frac{M_{2}(t)}{t} .
$$

Because of $w_{2}<0$, making use of Lemma $2, \lim _{t \rightarrow \infty} y(t)=0$, a.s. In this case, there is a sufficiently large constant $T_{2}\left(T_{2}>T_{1}\right)$ satisfying $-\varepsilon_{1} \leq y(t) \leq \varepsilon_{1}$, when $t \geq T_{2}$, a.s. Thus,

$$
\begin{aligned}
\frac{1}{t} \ln \frac{z(t)}{z_{0}} & \leq w_{3}+\beta \frac{1}{t} \int_{0}^{t} y(s) d s-\frac{1}{t} \int_{0}^{t} \frac{c_{3} z(s)}{x(s)+k_{2}} d s+\frac{\sigma_{3} B_{3}(t)}{t}+\frac{M_{3}(t)}{t} \\
& \leq w_{3}+\beta \frac{1}{t} \int_{0}^{t} \varepsilon_{1} d s-\frac{1}{t} \int_{0}^{t} \frac{c_{3} z(s)}{\varepsilon_{1}+k_{2}} d s+\frac{\sigma_{3} B_{3}(t)}{t}+\frac{M_{3}(t)}{t} \\
& \leq\left(w_{3}+\beta \varepsilon_{1}\right)-\frac{c_{3}}{\varepsilon_{1}+k_{2}} \frac{1}{t} \int_{0}^{t} z(s) d s+\frac{\sigma_{3} B_{3}(t)}{t}+\frac{M_{3}(t)}{t}
\end{aligned}
$$

Notice $0<\varepsilon_{1}<-\frac{w_{3}}{\beta}$, it follows from Lemma 2 that $\lim _{t \rightarrow \infty} z(t)=0$, a.s.

(ii) Similar to case (i), when $w_{1}<0, w_{2}<0$, it easily shows that $\lim _{t \rightarrow \infty} x(t)=0$, $\lim _{t \rightarrow \infty} y(t)=0$, and for an arbitrary small constant $\bar{\varepsilon}_{1}>0$,

$$
\frac{1}{t} \ln \frac{z(t)}{z_{0}} \leq\left(w_{3}+\beta \bar{\varepsilon}_{1}\right)-\frac{c_{3}}{\bar{\varepsilon}_{1}+k_{2}} \frac{1}{t} \int_{0}^{t} z(s) d s+\frac{\sigma_{3} B_{3}(t)}{t}+\frac{M_{3}(t)}{t} .
$$

Making use of Lemma 2, $w_{3}>0$ and the arbitrariness of $\bar{\varepsilon}_{1}, \limsup _{t \rightarrow \infty} \frac{1}{t} \int_{0}^{t} z(s) d s \leq \frac{w_{3} k_{2}}{c_{3}}$, a.s. is obviously verified. In contrast,

$$
\frac{1}{t} \ln \frac{z(t)}{z_{0}} \geq w_{3}-\frac{c_{3}}{k_{2}} \frac{1}{t} \int_{0}^{t} y(s) d s-\frac{c_{3}}{k_{2}} \frac{1}{t} \int_{0}^{t} z(s) d s+\frac{\sigma_{3} B_{3}(t)}{t}+\frac{M_{3}(t)}{t} .
$$

Noting that $\lim _{t \rightarrow \infty} y(t)=0$, a.s. and Lemma 2, we have

$$
\liminf _{t \rightarrow \infty} \frac{1}{t} \int_{0}^{t} z(s) d s \geq \frac{w_{3} k_{2}}{c_{3}}, \quad \text { a.s. }
$$

then we obtain $\lim _{t \rightarrow \infty} \frac{1}{t} \int_{0}^{t} z(s) d s=\frac{w_{3} k_{2}}{c_{3}}$, a.s.

(iii) It is easy to show that $\lim _{t \rightarrow \infty} x(t)=0$, a.s. when $w_{1}<0$. On the basis of (13) and Lemma 2, for $t>T_{1}$, $\limsup _{t \rightarrow \infty} \frac{1}{t} \int_{0}^{t} y(s) d s \leq \frac{w_{2}\left(\varepsilon_{1}+k_{2}\right)}{c_{2}}$, a.s. under the condition of $w_{2}>0$. Considering the arbitrariness of $\varepsilon_{1}, \lim \sup _{t \rightarrow \infty} \frac{1}{t} \int_{0}^{t} y(s) d s \leq \frac{w_{2} k_{2}}{c_{2}}$, a.s. Therefore, for an arbitrary constant $\varepsilon_{3}>0\left(0<\varepsilon_{3}<\frac{1}{\beta}\left|w_{3}+\frac{\beta k_{2}}{c_{2}} w_{2}\right|\right)$, there exists a number $T_{3}\left(T_{3}>T_{1}\right)$ such that

$$
\frac{1}{t} \int_{0}^{t} y(s) d s \leq \frac{w_{2} k_{2}}{c_{2}}+\varepsilon_{3}, \quad \text { a.s. }
$$


for $t>T_{3}$. Making use of (12), then for $t>T_{3}$, we have

$$
\begin{aligned}
\frac{1}{t} \ln \frac{z(t)}{z_{0}} & \leq w_{3}+\beta \frac{1}{t} \int_{0}^{t} y(s) d s-\frac{1}{t} \int_{0}^{t} \frac{c_{3} z(s)}{\varepsilon_{1}+k_{2}} d s+\frac{\sigma_{3} B_{3}(t)}{t}+\frac{M_{3}(t)}{t} \\
& \leq w_{3}+\beta\left(\frac{w_{2} k_{2}}{c_{2}}+\varepsilon_{3}\right)-\frac{c_{3}}{\varepsilon_{1}+k_{2}} \frac{1}{t} \int_{0}^{t} z(s) d s+\frac{\sigma_{3} B_{3}(t)}{t}+\frac{M_{3}(t)}{t} .
\end{aligned}
$$

(a) If $w_{3}+\frac{\beta k_{2}}{c_{2}} w_{2}<0$, then

$$
\lim _{t \rightarrow \infty} z(t)=0, \quad \text { a.s. }
$$

For the susceptible predator population,

$$
\frac{1}{t} \ln \frac{y(t)}{y_{0}} \geq w_{2}-\frac{1}{t} \int_{0}^{t} \frac{c_{2} y(s)}{k_{2}} d s-\frac{1}{t} \int_{0}^{t} \frac{c_{2} z(s)}{k_{2}} d s-\beta \frac{1}{t} \int_{0}^{t} z(s) d s+\frac{\sigma_{2} B_{2}(t)}{t}+\frac{M_{2}(t)}{t} .
$$

Because of (9), (10), (14) and Lemma 2, we have $\liminf _{t \rightarrow \infty} \frac{1}{t} \int_{0}^{t} y(s) d s \geq \frac{w_{2} k_{2}}{c_{2}}$, a.s. Then $\lim _{t \rightarrow \infty} \frac{1}{t} \int_{0}^{t} y(s) d s=\frac{w_{2} k_{2}}{c_{2}}$, a.s.

(b) If $w_{3}+\frac{\beta k_{2}}{c_{2}} w_{2}>0$, by virtue of Lemma 2 and the arbitrariness of $\varepsilon_{1}$ and $\varepsilon_{3}$, we have

$$
\limsup _{t \rightarrow \infty} \frac{1}{t} \int_{0}^{t} z(s) d s \leq \frac{\left(w_{3} c_{2}+\beta k_{2} w_{2}\right) k_{2}}{c_{2} c_{3}}, \quad \text { a.s. }
$$

Then

$$
\frac{1}{t} \ln \frac{y(t)}{y_{0}} \geq w_{2}-\frac{c_{2}}{k_{2}} \frac{1}{t} \int_{0}^{t} y(s) d s-\left(\frac{c_{2}}{k_{2}}+\beta\right) \frac{1}{t} \int_{0}^{t} z(s) d s+\frac{\sigma_{2} B_{2}(t)}{t}+\frac{M_{2}(t)}{t} .
$$

Notice (15), for sufficiently large $t, \frac{1}{t} \int_{0}^{t} z(s) d s \leq \frac{\left(w_{3} c_{2}+\beta k_{2} w_{2}\right) k_{2}}{c_{2} c_{3}}+\varepsilon_{4}$, a.s., where $\varepsilon_{4}$ is an arbitrary positive number. Hence, by (9), (10), Lemma 2 and the arbitrariness of $\varepsilon_{4}$, we have

$$
\liminf _{t \rightarrow \infty} \frac{1}{t} \int_{0}^{t} y(s) d s \geq \frac{k_{2}}{c_{2}}\left[w_{2}-\frac{\left(c_{2}+k_{2} \beta\right)\left(w_{3} c_{2}+\beta k_{2} w_{2}\right)}{c_{2} c_{3}}\right]
$$

when $w_{2}>\frac{\left(c_{2}+k_{2} \beta\right)\left(w_{3} c_{2}+\beta k_{2} w_{2}\right)}{c_{2} c_{3}}$. Here we define $Q=\frac{k_{2}}{c_{2}}\left[w_{2}-\frac{\left(c_{2}+k_{2} \beta\right)\left(w_{3} c_{2}+\beta k_{2} w_{2}\right)}{c_{2} c_{3}}\right]$.

In this case,

$$
\frac{1}{t} \ln \frac{z(t)}{z_{0}} \geq w_{3}+\beta \frac{1}{t} \int_{0}^{t} y(s) d s-\frac{c_{3}}{k_{2}} \frac{1}{t} \int_{0}^{t} y(s) d s-\frac{c_{3}}{k_{2}} \frac{1}{t} \int_{0}^{t} z(s) d s+\frac{\sigma_{3} B_{3}(t)}{t}+\frac{M_{3}(t)}{t} .
$$

For sufficiently large $t$, we have

$$
\frac{1}{t} \ln \frac{z(t)}{z_{0}} \geq\left[w_{3}+\beta Q-\frac{c_{3}}{k_{2}} \frac{w_{2} k_{2}}{c_{2}}\right]-\frac{c_{3}}{k_{2}} \frac{1}{t} \int_{0}^{t} z(s) d s+\frac{\sigma_{3} B_{3}(t)}{t}+\frac{M_{3}(t)}{t} .
$$

If $w_{3}+\beta Q>\frac{c_{3} w_{2}}{c_{2}}$, then by Lemma 2 , we get

$$
\liminf _{t \rightarrow \infty} \frac{1}{t} \int_{0}^{t} z(s) d s \geq \frac{\left[w_{3}+\beta Q-\frac{c_{3} w_{2}}{c_{2}}\right] k_{2}}{c_{3}}, \quad \text { a.s. }
$$


(iv) According to (11), we have

$$
\frac{1}{t} \ln \frac{x(t)}{x_{0}} \leq w_{1}-b_{1} \frac{1}{t} \int_{0}^{t} x(s) d s+\frac{\sigma_{1} B_{1}(t)}{t}+\frac{M_{1}(t)}{t}
$$

Making use of Lemma 2, (9) and (10), we have

$$
\limsup _{t \rightarrow \infty} \frac{1}{t} \int_{0}^{t} x(s) d s \leq \frac{w_{1}}{b_{1}}, \quad \text { a.s. }
$$

and

$$
\frac{1}{t} \ln \frac{y(t)}{y_{0}} \leq w_{2}+\frac{\sigma_{2} B_{2}(t)}{t}+\frac{M_{2}(t)}{t} .
$$

Because of $w_{2}<0$ and (9), (10), we get $\lim \sup _{t \rightarrow \infty} \frac{\ln y(t)}{t}<0$, a.s., namely

$$
\lim _{t \rightarrow \infty} y(t)=0, \quad \text { a.s. }
$$

Then

$$
\frac{1}{t} \ln \frac{z(t)}{z_{0}} \leq w_{3}+\beta \frac{1}{t} \int_{0}^{t} y(s) d s+\frac{\sigma_{3} B_{3}(t)}{t}+\frac{M_{3}(t)}{t} .
$$

Considering (9), (10), (17) and $w_{3}<0$, we have $\lim \sup _{t \rightarrow \infty} \frac{\ln z(t)}{t}<0$; therefore,

$$
\lim _{t \rightarrow \infty} z(t)=0 \text {, a.s. }
$$

At this time, for sufficiently large $t$,

$$
\frac{1}{t} \ln \frac{x(t)}{x_{0}} \geq w_{1}-b_{1} \frac{1}{t} \int_{0}^{t} x(s) d s-\frac{c_{1}}{k_{1}} \frac{1}{t} \int_{0}^{t} y(s) d s-\frac{\tilde{p} c_{1}}{k_{1}} \frac{1}{t} \int_{0}^{t} z(s) d s+\frac{\sigma_{1} B_{1}(t)}{t}+\frac{M_{1}(t)}{t} .
$$

By (17), (18) and Lemma 2,

$$
\liminf _{t \rightarrow \infty} \frac{1}{t} \int_{0}^{t} x(s) d s \geq \frac{w_{1}}{b_{1}}, \quad \text { a.s. }
$$

Combining (19) with (16), we have $\lim _{t \rightarrow \infty} \frac{1}{t} \int_{0}^{t} x(s) d s=\frac{w_{1}}{b_{1}}$, a.s.

(v) It is easy to have $\lim \sup _{t \rightarrow \infty} \frac{1}{t} \int_{0}^{t} x(s) d s \leq \frac{w_{1}}{b_{1}}$, a.s. when $w_{1}>0$ and

$$
\begin{aligned}
& \frac{1}{t} \ln \frac{y(t)}{y_{0}} \geq w_{2}-\frac{c_{2}}{k_{2}} \frac{1}{t} \int_{0}^{t} y(s) d s-\left(\frac{c_{2}}{k_{2}}+\beta\right) \frac{1}{t} \int_{0}^{t} z(s) d s+\frac{\sigma_{2} B_{2}(t)}{t}+\frac{M_{2}(t)}{t}, \\
& \frac{1}{t} \ln \frac{z(t)}{z_{0}} \geq w_{3}-\frac{c_{3}}{k_{2}} \frac{1}{t} \int_{0}^{t} y(s) d s-\frac{c_{3}}{k_{2}} \frac{1}{t} \int_{0}^{t} z(s) d s+\frac{\sigma_{3} B_{3}(t)}{t}+\frac{M_{3}(t)}{t} .
\end{aligned}
$$

Then

$$
\begin{aligned}
\frac{1}{t} \ln \frac{y(t)}{y_{0}}+\frac{1}{t} \ln \frac{z(t)}{z_{0}} \geq & \left(w_{2}+w_{3}\right)-\frac{c_{2}+c_{3}}{k_{2}} \frac{1}{t} \int_{0}^{t} y(s) d s-\frac{c_{2}+c_{3}+k_{2} \beta}{k_{2}} \frac{1}{t} \int_{0}^{t} z(s) d s \\
& +\sum_{i=2}^{3}\left[\frac{\sigma_{i} B_{i}(t)}{t}+\frac{M_{i}(t)}{t}\right],
\end{aligned}
$$


namely

$$
\begin{aligned}
\frac{1}{t} \ln (y(t) z(t))-\frac{1}{t} \ln \left(y_{0} z_{0}\right) \geq & \left(w_{2}+w_{3}\right)-\frac{c_{2}+c_{3}+k_{2} \beta}{k_{2}} \frac{1}{t} \int_{0}^{t}[y(s)+z(s)] d s \\
& +\sum_{i=2}^{3}\left[\frac{\sigma_{i} B_{i}(t)}{t}+\frac{M_{i}(t)}{t}\right] .
\end{aligned}
$$

By virtue of the formula $(a+b)^{2} \geq 2 a b$ for $a, b \in R_{+}$, we can get $2 \ln (a+b) \geq \ln 2+\ln (a b)$, hence

$$
\begin{aligned}
\frac{2 \ln (y(t)+z(t))}{t}-\frac{\ln 2}{t}-\frac{\ln \left(y_{0} z_{0}\right)}{t} \geq & \left(w_{2}+w_{3}\right)-\frac{\left(c_{2}+c_{3}+k_{2} \beta\right)}{k_{2}} \frac{1}{t} \int_{0}^{t}[y(s)+z(s)] d s \\
& +\sum_{i=2}^{3}\left[\frac{\sigma_{i} B_{i}(t)}{t}+\frac{M_{i}(t)}{t}\right]
\end{aligned}
$$

then

$$
\begin{aligned}
\frac{\ln (y(t)+z(t))}{t}-\frac{\ln 2}{2 t}-\frac{\ln \left(y_{0} z_{0}\right)}{2 t} \geq & \frac{\left(w_{2}+w_{3}\right)}{2}-\frac{\left(c_{2}+c_{3}+k_{2} \beta\right)}{2 k_{2}} \frac{1}{t} \int_{0}^{t}[y(s)+z(s)] d s \\
& +\frac{1}{2} \sum_{i=2}^{3}\left[\frac{\sigma_{i} B_{i}(t)}{t}+\frac{M_{i}(t)}{t}\right] .
\end{aligned}
$$

By Lemma 2, we get

$$
\liminf _{t \rightarrow \infty} \frac{1}{t} \int_{0}^{t}[y(s)+z(s)] d s \geq \frac{\left(w_{2}+w_{3}\right) k_{2}}{c_{2}+c_{3}+k_{2} \beta}, \quad \text { a.s. }
$$

In this case, considering $0<\tilde{p}<1$, we have

$$
\frac{1}{t} \ln \frac{x(t)}{x_{0}} \geq w_{1}-b_{1} \frac{1}{t} \int_{0}^{t} x(s) d s-\frac{c_{1}}{k_{1}} \frac{1}{t} \int_{0}^{t}[y(s)+z(s)] d s+\frac{\sigma_{1} B_{1}(t)}{t}+\frac{M_{1}(t)}{t} .
$$

Taking the inferior limit, by virtue of Lemma 1 and (9), (10), we get

$$
\begin{aligned}
0 \geq & \limsup _{t \rightarrow \infty} \frac{1}{t} \ln \frac{x(t)}{x_{0}} \geq \liminf _{t \rightarrow \infty} \frac{1}{t} \ln \frac{x(t)}{x_{0}} \\
\geq & w_{1}-b_{1} \liminf _{t \rightarrow \infty}\left[\frac{1}{t} \int_{0}^{t} x(s) d s\right]-\frac{c_{1}}{k_{1}} \liminf _{t \rightarrow \infty}\left[\frac{1}{t} \int_{0}^{t}[y(s)+z(s)] d s\right] \\
& +\liminf _{t \rightarrow \infty}\left[\frac{\sigma_{1} B_{1}(t)}{t}+\frac{M_{1}(t)}{t}\right] .
\end{aligned}
$$

Hence,

$$
\liminf _{t \rightarrow \infty}\left[\frac{1}{t} \int_{0}^{t} x(s) d s\right] \geq \frac{w_{1}}{b_{1}}-\frac{\left(w_{2}+w_{3}\right) k_{2} c_{1}}{b_{1} k_{1}\left(c_{2}+c_{3}+k_{2} \beta\right)}, \quad \text { a.s. }
$$

\section{Stochastically ultimate boundedness}

From the biological point of view, the nonexplosion and positiveness property of solution in a population dynamical system is not enough. In this section we will show that the $p$ th moment of solution (3) is bounded. 
Definition 2 The solution $X(t)$ of system (3) is called stochastically ultimate bounded if, for any $\epsilon \in(0,1)$, there is a constant $H:=H(\epsilon)$ such that, for any $X(0) \in R_{+}^{3}$,

$$
\limsup _{t \rightarrow \infty} P\{|X(t)| \leq H\} \geq 1-\epsilon
$$

Theorem 3 Assume $(x(t), y(t), z(t))^{T}$ to be a solution to system (3) with the initial value $\left(x_{0}, y_{0}, z_{0}\right) \in R_{+}^{3}$. If $k_{2}>\frac{\left(1+a_{1}\right)^{2}}{4 b_{1}}$ for all $p>0$, there exist constants $\bar{K}_{i}(p)(i=1,2,3)$ such that

$$
\limsup _{t \rightarrow \infty} E\left[x^{p}(t)\right] \leq \bar{K}_{1}(p), \quad \quad \limsup _{t \rightarrow \infty} E\left[y^{p}(t)\right] \leq \bar{K}_{2}(p), \quad \limsup _{t \rightarrow \infty} E\left[z^{p}(t)\right] \leq \bar{K}_{3}(p) .
$$

Proof Making use of Itô's formula and then taking expectations yield

$$
\begin{aligned}
d\left(e^{t} x^{p}(t)\right)= & e^{t} x^{p}(t)\left\{1+p\left[a_{1}-b_{1} x-\frac{c_{1} y}{x+k_{1}}-\frac{\tilde{p} c_{1} z}{x+k_{1}}\right]+\frac{p(p-1)}{2} \sigma_{1}^{2}\right. \\
& \left.+\int_{\mathbb{Y}}\left[\left(1+\gamma_{1}(u)\right)^{p}-1-p \gamma_{1}(u)\right] \lambda(d u)\right\} d t+p \sigma_{1} e^{t} x^{p}(t) d B_{1}(t) \\
& +\int_{\mathbb{Y}} e^{t} x^{p}(t)\left[\left(1+\gamma_{1}(u)\right)^{p}-1\right] \tilde{N}(d t, d u), \\
E\left(e^{t} x^{p}(t)\right)= & x^{p}(0)+p E \int_{0}^{t} e^{s} x^{p}(s)\left\{\frac{1}{p}+a_{1}+\frac{(p-1)}{2} \sigma_{1}^{2}\right. \\
& +\frac{1}{p} \int_{\mathbb{Y}}\left[\left(1+\gamma_{1}(u)\right)^{p}-1-p \gamma_{1}(u)\right] \lambda(d u) \\
& \left.-b_{1} x(s)-\frac{c_{1} y(s)}{x(s)+k_{1}}-\frac{\tilde{p} c_{1} z(s)}{x(s)+k_{1}}\right\} d s \\
\leq & x^{p}(0)+p E \int_{0}^{t} e^{s} x^{p}(s)\left\{\frac{1}{p}+a_{1}+\frac{(p-1)}{2} \sigma_{1}^{2}\right. \\
& \left.+\frac{1}{p} \int_{\mathbb{Y}}\left[\left(1+\gamma_{1}(u)\right)^{p}-1-p \gamma_{1}(u)\right] \lambda(d u)-b_{1} x(s)\right\} d s .
\end{aligned}
$$

Note for a function $f(v)=v^{p}(a-b v), v \in[0,+\infty), p>0, a>0, b>0, f(v)$ has a maximum value at $\frac{a p}{(p+1) b}$, namely

$$
f(v) \leq f\left(\frac{a p}{(p+1) b}\right)=\frac{a^{p+1} p^{p}}{(p+1)^{p+1} b^{p}} .
$$

Therefore,

$$
E\left(e^{t} x^{p}(t)\right) \leq x^{p}(0)+E \int_{0}^{t} e^{s} \bar{K}_{1}(p) d s \leq x^{p}(0)+\bar{K}_{1}(p)\left(e^{t}-1\right),
$$

where

$$
\bar{K}_{1}(p)=\frac{\left[1+a_{1} p+\frac{p(p-1)}{2} \sigma_{1}^{2}+\int_{\mathbb{Y}}\left[\left(1+\gamma_{1}(u)\right)^{p}-1-p \gamma_{1}(u)\right] \lambda(d u)\right]^{p+1}}{(p+1)^{p+1} b_{1}^{p}} .
$$

Let $t \rightarrow+\infty$, we have

$$
\limsup _{t \rightarrow \infty} E\left[x^{p}(t)\right] \leq \bar{K}_{1}(p), \quad \text { a.s. }
$$


Similarly, we can compute

$$
\begin{aligned}
d\left(e^{t} y^{p}(t)\right)= & e^{t} y^{p}(t)\left\{1+p\left[a_{2}-\frac{c_{2}(y+z)}{x+k_{2}}-\beta z\right]+\frac{p(p-1)}{2} \sigma_{2}^{2}\right. \\
& \left.+\int_{\mathbb{Y}}\left[\left(1+\gamma_{2}(u)\right)^{p}-p \gamma_{2}(u)-1\right] \lambda(d u)\right\} d t+p \sigma_{2} e^{t} y^{p}(t) d B_{2}(t) \\
& +\int_{\mathbb{Y}} e^{t} y^{p}(t)\left[\left(1+\gamma_{2}(u)\right)^{p}-1\right] \tilde{N}(d t, d u) .
\end{aligned}
$$

Thus, taking expectations leads to

$$
\begin{aligned}
E\left(e^{t} y^{p}(t)\right)= & y^{p}(0)+E \int_{0}^{t} e^{s} y^{p}(s)\left[1+p a_{2}+\frac{p(p-1)}{2} \sigma_{2}^{2}\right. \\
& +\int_{\mathbb{Y}}\left[\left(1+\gamma_{2}(u)\right)^{p}-p \gamma_{2}(u)-1\right] \lambda(d u) \\
& \left.-\frac{c_{2} p(y(s)+z(s))}{x(s)+k_{2}}-p \beta z(s)\right] d s \\
\leq & y^{p}(0)+E \int_{0}^{t} e^{s} y^{p}(s)\left[1+p a_{2}+\frac{p(p-1)}{2} \sigma_{2}^{2}\right. \\
& +\int_{\mathbb{Y}}\left[\left(1+\gamma_{2}(u)\right)^{p}-p \gamma_{2}(u)-1\right] \lambda(d u) \\
& \left.-\frac{c_{2} p y(s)}{k_{2}}+\frac{c_{2} p x(s) y(s)}{k_{2}\left(x(s)+k_{2}\right)}\right] d s .
\end{aligned}
$$

By virtue of (21), for arbitrary small $\varepsilon_{0}>0\left(\varepsilon_{0}<k_{2}-\bar{K}_{1}(1)\right)$, there exists a positive constant $T_{0}$ satisfying

$$
E\left(x^{p}(t)\right) \leq \bar{K}_{1}(p)+\varepsilon_{0}
$$

for $t>T_{0}$. Therefore,

$$
\begin{aligned}
E\left(e^{t} y^{p}(t)\right) \leq & y^{p}(0)+\int_{0}^{t} e^{s} E\left\{y ^ { p } ( s ) \left[1+p a_{2}+\frac{p(p-1)}{2} \sigma_{2}^{2}+\int_{\mathbb{Y}}\left[\left(1+\gamma_{2}(u)\right)^{p}\right.\right.\right. \\
& \left.\left.\left.-p \gamma_{2}(u)-1\right] \lambda(d u)-\frac{c_{2} p y(s)}{k_{2}}\right]\right\} d s+\frac{c_{2} p}{k_{2}^{2}} \int_{0}^{t} e^{s} E(x(s)) E\left(y^{p+1}(s)\right) d s \\
\leq & y^{p}(0)+\int_{0}^{t} e^{s} E\left\{y ^ { p } ( s ) \left[1+p a_{2}+\frac{p(p-1)}{2} \sigma_{2}^{2}+\int_{\mathbb{Y}}\left[\left(1+\gamma_{2}(u)\right)^{p}\right.\right.\right. \\
& \left.\left.\left.-p \gamma_{2}(u)-1\right] \lambda(d u)-\frac{c_{2} p y(s)}{k_{2}}\right]\right\} d s+\frac{c_{2} p}{k_{2}^{2}}\left(\bar{K}_{1}(1)+\varepsilon_{0}\right) \int_{0}^{t} e^{s} E\left(y^{p+1}(s)\right) d s \\
\leq & y^{p}(0)+\int_{0}^{t} e^{s} E\left\{y ^ { p } ( s ) \left[1+p a_{2}+\frac{p(p-1)}{2} \sigma_{2}^{2}+\int_{\mathbb{Y}}\left[\left(1+\gamma_{2}(u)\right)^{p}\right.\right.\right. \\
& \left.\left.\left.-p \gamma_{2}(u)-1\right] \lambda(d u)-\frac{c_{2} p y(s)}{k_{2}}+\frac{c_{2} p}{k_{2}^{2}}\left(\bar{K}_{1}(1)+\varepsilon_{0}\right) y(s)\right]\right\} d s .
\end{aligned}
$$


If $k_{2}>\bar{K}_{1}(1)$, then $k_{2}>\bar{K}_{1}(1)+\varepsilon_{0}$. According to (20), the following result is available:

$$
E\left(e^{t} y^{p}(t)\right) \leq y^{p}(0)+\int_{0}^{t} e^{s} K_{2}(p) d s=y^{p}(0)+K_{2}(p)\left(e^{t}-1\right) .
$$

Here

$$
\begin{aligned}
K_{2}(p)= & \frac{\left[1+p a_{2}+\frac{p(p-1)}{2} \sigma_{2}^{2}+\int_{\mathbb{Y}}\left[\left(1+\gamma_{2}(u)\right)^{p}-p \gamma_{2}(u)-1\right] \lambda(d u)\right]^{p+1}}{(p+1)^{p+1}} \\
& \cdot \frac{k_{2}^{2 p}}{c_{2}^{p}\left(k_{2}-\bar{K}_{1}(1)-\varepsilon_{0}\right)^{p}},
\end{aligned}
$$

where $\bar{K}_{1}(1)=\frac{\left(1+a_{1}\right)^{2}}{4 b_{1}}$. Similarly, $\lim \sup _{t \rightarrow \infty} E\left[y^{p}(t)\right] \leq K_{2}(p)$, here because of the arbitrariness of $\varepsilon_{0}$, define

$$
\bar{K}_{2}(p)=\frac{\left[1+p a_{2}+\frac{p(p-1)}{2} \sigma_{2}^{2}+\int_{\mathbb{Y}}\left[\left(1+\gamma_{2}(u)\right)^{p}-p \gamma_{2}(u)-1\right] \lambda(d u)\right]^{p+1}}{(p+1)^{p+1}} \cdot \frac{k_{2}^{2 p}}{c_{2}^{p}\left(k_{2}-\bar{K}_{1}(1)\right)^{p}},
$$

thus,

$$
\limsup _{t \rightarrow \infty} E\left[y^{p}(t)\right] \leq \bar{K}_{2}(p) .
$$

And

$$
\begin{aligned}
d\left(e^{t} z^{p}(t)\right)= & e^{t} z^{p}(t)\left\{1+p\left[\beta y+a_{3}-\frac{c_{3}(y+z)}{x+k_{2}}\right]+\frac{p(p-1)}{2} \sigma_{3}^{2}\right. \\
& \left.+\int_{\mathbb{Y}}\left[\left(1+\gamma_{3}(u)\right)^{p}-p \gamma_{3}(u)-1\right] \lambda(d u)\right\} d t+p \sigma_{3} e^{t} z^{p}(t) d B_{3}(t) \\
& +\int_{\mathbb{Y}} e^{t} z^{p}(t)\left[\left(1+\gamma_{3}(u)\right)^{p}-1\right] \tilde{N}(d t, d u), \\
E\left(e^{t} z^{p}(t)\right)= & z^{p}(0)+E \int_{0}^{t} e^{s} z^{p}(s)\left[1+p a_{3}+\frac{p(p-1)}{2} \sigma_{3}^{2}\right. \\
& +\int_{\mathbb{Y}}\left[\left(1+\gamma_{3}(u)\right)^{p}-p \gamma_{3}(u)-1\right] \lambda(d u) \\
& \left.+p \beta y(s)-\frac{c_{3} p(y(s)+z(s))}{x(s)+k_{2}}\right] d s \\
\leq & z^{p}(0)+E \int_{0}^{t} e^{s} z^{p}(s)\left[1+p a_{3}+\frac{p(p-1)}{2} \sigma_{3}^{2}\right. \\
& +\int_{\mathbb{Y}}\left[\left(1+\gamma_{3}(u)\right)^{p}-p \gamma_{3}(u)-1\right] \lambda(d u) \\
& \left.+p \beta y(s)-\frac{c_{3} p z(s)}{x(s)+k_{2}}\right] d s \\
\leq & z^{p}(0)+E \int_{0}^{t} p \beta e^{s} z^{p}(s) y(s) d s+E \int_{0}^{t} e^{s} z^{p}(s)\left[1+p a_{3}+\frac{p(p-1)}{2} \sigma_{3}^{2}\right. \\
& \left.+\int_{\mathbb{Y}}\left[\left(1+\gamma_{3}(u)\right)^{p}-p \gamma_{3}(u)-1\right] \lambda(d u)-\frac{c_{3} p z(s)}{k_{2}}+\frac{c_{3} p x(s) z(s)}{k_{2}\left(x(s)+k_{2}\right)}\right] d s .
\end{aligned}
$$


On the basis of Young's inequality, we have

$$
\begin{aligned}
y(t) z^{p}(t) & \leq \frac{y^{p+1}(t)}{p+1}+\frac{p}{p+1}\left(z^{p}(t)\right)^{\frac{p+1}{p}} \\
& \leq \frac{y^{p+1}(t)}{p+1}+\frac{p}{p+1} z^{p+1}(t) .
\end{aligned}
$$

Notice $\frac{p}{p+1}<1$, we have $y(t) z^{p}(t) \leq \frac{y^{p+1}(t)}{p+1}+z^{p+1}(t)$, then

$$
\begin{aligned}
E\left(e^{t} z^{p}(t)\right) \leq & z^{p}(0)+p \beta E \int_{0}^{t} e^{s} z^{p+1}(s) d s+p \beta E \int_{0}^{t} e^{s} \frac{1}{p+1} y^{p+1}(s) d s \\
& +E \int_{0}^{t} e^{s} z^{p}(s)\left[1+p a_{3}+\frac{p(p-1)}{2} \sigma_{3}^{2}\right. \\
& \left.+\int_{\mathbb{Y}}\left[\left(1+\gamma_{3}(u)\right)^{p}-p \gamma_{3}(u)-1\right] \lambda(d u)-\frac{c_{3} p z(s)}{k_{2}}+\frac{c_{3} p x(s) z(s)}{k_{2}^{2}}\right] d s \\
\leq & z^{p}(0)+\frac{p \beta}{p+1} E \int_{0}^{t} e^{s} y^{p+1}(s) d s+E \int_{0}^{t} e^{s} z^{p}(s)\left[1+p a_{3}+\frac{p(p-1)}{2} \sigma_{3}^{2}\right. \\
& \left.+\int_{\mathbb{Y}}\left[\left(1+\gamma_{3}(u)\right)^{p}-p \gamma_{3}(u)-1\right] \lambda(d u)-\left(\frac{c_{3} p}{k_{2}}-p \beta\right) z(s)\right] d s \\
& +\frac{c_{3} p}{k_{2}^{2}} \int_{0}^{t} e^{s} E(x(s)) E\left(z^{p+1}(s)\right) d s .
\end{aligned}
$$

Considering (22), we can see that there exist positive constants $\vec{K}_{2}(p)$ and $\vec{T}_{2}\left(\vec{T}_{2}>T_{0}\right)$, for $t>\vec{T}_{2}$, one can obtain that $E\left[y^{p}(t)\right] \leq \vec{K}_{2}(p)$. Then, for $t>\vec{T}_{2}$,

$$
\begin{aligned}
E\left(e^{t} z^{p}(t)\right) \leq & z^{p}(0)+\frac{p \beta}{p+1} \vec{K}_{2}(p+1)\left[e^{t}-1\right]+\int_{0}^{t} e^{s} E\left\{z ^ { p } ( s ) \left[1+p a_{3}+\frac{p(p-1)}{2} \sigma_{3}^{2}\right.\right. \\
& +\int_{\mathbb{Y}}\left[\left(1+\gamma_{3}(u)\right)^{p}-p \gamma_{3}(u)-1\right] \lambda(d u) \\
& \left.\left.-\left(\frac{c_{3} p}{k_{2}}-p \beta-\frac{c_{3} p}{k_{2}^{2}}\left(\bar{K}_{1}(1)+\varepsilon_{0}\right)\right) z(s)\right]\right\} d s \\
\leq & z^{p}(0)+\frac{p \beta}{p+1} \vec{K}_{2}(p+1)\left[e^{t}-1\right]+\int_{0}^{t} e^{s} K_{3}(p) d s \\
\leq & z^{p}(0)+\frac{p \beta}{p+1} \vec{K}_{2}(p+1)\left[e^{t}-1\right]+K_{3}(p)\left[e^{t}-1\right]
\end{aligned}
$$

where

$$
\begin{aligned}
K_{3}(p)= & \frac{\left[1+p a_{3}+\frac{p(p-1)}{2} \sigma_{3}^{2}+\int_{\mathbb{Y}}\left[\left(1+\gamma_{3}(u)\right)^{p}-p \gamma_{3}(u)-1\right] \lambda(d u)\right]^{p+1}}{(p+1)^{p+1}} \\
& \cdot \frac{p^{p}}{\left[\frac{c_{3} p}{k_{2}}-p \beta-\frac{c_{3} p}{k_{2}^{2}}\left(\bar{K}_{1}(1)+\varepsilon_{0}\right)\right]^{p}} .
\end{aligned}
$$

Take the superior limit results in

$$
\limsup _{t \rightarrow \infty} E\left[z^{p}(t)\right] \leq \frac{p \beta}{p+1} \vec{K}_{2}(p+1)+K_{3}(p) .
$$


Notice the arbitrariness of $\varepsilon_{0}$, let

$$
\begin{aligned}
\bar{K}_{3}(p)= & \frac{p \beta}{p+1} \vec{K}_{2}(p+1)+\frac{\left[1+p a_{3}+\frac{p(p-1)}{2} \sigma_{3}^{2}+\int_{\mathbb{Y}}\left[\left(1+\gamma_{3}(u)\right)^{p}-p \gamma_{3}(u)-1\right] \lambda(d u)\right]^{p+1}}{(p+1)^{p+1}} \\
& \cdot \frac{p^{p}}{\left[\frac{c_{3} p}{k_{2}}-p \beta-\frac{c_{3} p}{k_{2}^{2}} \bar{K}_{1}(1)\right]^{p}},
\end{aligned}
$$

clearly,

$$
\limsup _{t \rightarrow \infty} E\left[z^{p}(t)\right] \leq \bar{K}_{3}(p)
$$

thus the proof of Theorem 3 is completed.

By virtue of the formula of Lemma 2.1 in [10], we have

$$
n^{\left(1-\frac{p}{2}\right) \wedge 0}|x|^{p} \leq \sum_{i=1}^{n} x_{i}^{p} \leq n^{\left(1-\frac{p}{2}\right) \vee 0}|x|^{p}
$$

for $\forall p>0, x \in R_{+}^{n}$, where $R_{+}^{n}:=\left\{x \in R^{n}, x_{i}>0,1 \leq i \leq n\right\}$.

For solution $X(t)=(x(t), y(t), z(t))^{T} \in R_{+}^{3}$ and $p>0$, we have

$$
3^{\left(1-\frac{p}{2}\right) \wedge 0}|X(t)|^{p} \leq x^{p}(t)+y^{p}(t)+z^{p}(t) .
$$

By Theorem 3,

$$
\limsup _{t \rightarrow \infty} E[X(t)]^{p} \leq\left(\frac{1}{3}\right)^{\left(1-\frac{p}{2}\right) \wedge 0} \limsup _{t \rightarrow \infty} E\left[x^{p}(t)+y^{p}(t)+z^{p}(t)\right] \leq\left(\frac{1}{3}\right)^{\left(1-\frac{p}{2}\right) \wedge 0} \sum_{i=1}^{3} \bar{K}_{i}(p) .
$$

As an application of Theorem 3, together with the Chebyshev inequality, we can also establish the following corollary.

Corollary 1 Under conditions of Theorem 3, the solution of system (3) is stochastically ultimate bounded.

\section{Numerical simulation}

In this section, we give some examples and numerical simulations to illustrate our analytical findings. These numerical simulations are given by the Euler scheme of [20].

For system (3), we choose the initial value $\left(x_{0}, y_{0}, z_{0}\right)=(0.2,0.1,0.2)$, and

$$
\begin{array}{llll}
b_{1}=0.3, & c_{1}=0.4, & \tilde{p}=0.1, & c_{2}=0.6, \quad k_{1}=0.5, \\
k_{2}=0.5, & \beta=0.4, & c_{3}=0.5, & \sigma_{i}=0.5 \quad(i=1,2,3),
\end{array}
$$

$\mathbb{Y}=(0,+\infty), \lambda(\mathbb{Y})=1$. In the following, we change the values of part parameters to observe the asymptotic behavior of solution for system (3). 


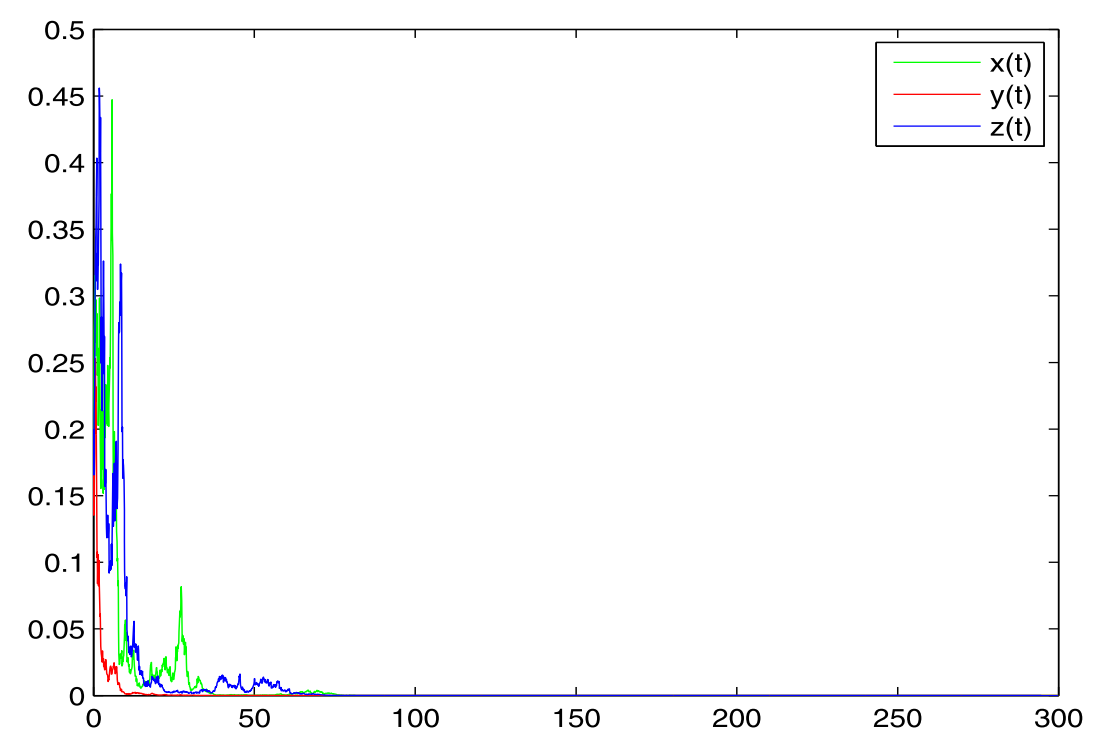

Figure 1 Solutions of system (3) for $a_{1}=0.02, a_{2}=0.03, a_{3}=0.02, \gamma_{1}(u)=\gamma_{3}(u)=0.2, \gamma_{2}(u)=0.4$, the other parameters are the same as in (23), with step size $\Delta t=0.1>0$

(1) In Figure 1, let $a_{1}=0.02, a_{2}=0.03, a_{3}=0.02, \gamma_{1}(u)=\gamma_{3}(u)=0.2, \gamma_{2}(u)=0.4$, the other parameters are the same as above, then

$$
\begin{aligned}
& w_{1}=a_{1}-\frac{\sigma_{1}^{2}}{2}-\int_{\mathbb{Y}}\left[\gamma_{1}(u)-\ln \left(1+\gamma_{1}(u)\right)\right] \lambda(d u)=-0.1227<0, \\
& w_{2}=a_{2}-\frac{\sigma_{2}^{2}}{2}-\int_{\mathbb{Y}}\left[\gamma_{2}(u)-\ln \left(1+\gamma_{2}(u)\right)\right] \lambda(d u)=-0.1585<0, \\
& w_{3}=a_{3}-\frac{\sigma_{3}^{2}}{2}-\int_{\mathbb{Y}}\left[\gamma_{3}(u)-\ln \left(1+\gamma_{3}(u)\right)\right] \lambda(d u)=-0.1227<0 .
\end{aligned}
$$

Thus, in accordance with case (i) in Theorem 2, all the species will go to extinction, Figure 1 confirms it.

(2) In Figure 2, let $a_{1}=0.02, a_{2}=0.03, a_{3}=0.5, \gamma_{1}(u)=\gamma_{2}(u)=0.2, \gamma_{3}(u)=0.4$, the other parameters remain the same as in (23), then

$$
w_{1}=-0.1227<0, \quad w_{2}=-0.1127<0, \quad w_{3}=0.3115>0 .
$$

By the case (ii) of Theorem 2, the prey population and the susceptible predator population will be extinct, the infected predator population will be stable in the mean. Figure 2 shows it.

(3) In Figure 3, we assume $a_{1}=0.06, a_{2}=0.6, a_{3}=0.2, \sigma_{3}=0.8, \beta=k_{2}=0.2, \gamma_{1}(u)=0.4$, $\gamma_{2}(u)=\gamma_{3}(u)=0.2$, the other parameters are the same as in (23), at this time

$$
\begin{aligned}
& w_{1}=-0.1285<0, \quad w_{2}=0.4573>0, \\
& w_{3}=-0.1377<0, \quad w_{3}+\frac{\beta k_{2}}{c_{2}} w_{2}=-0.1072<0 .
\end{aligned}
$$




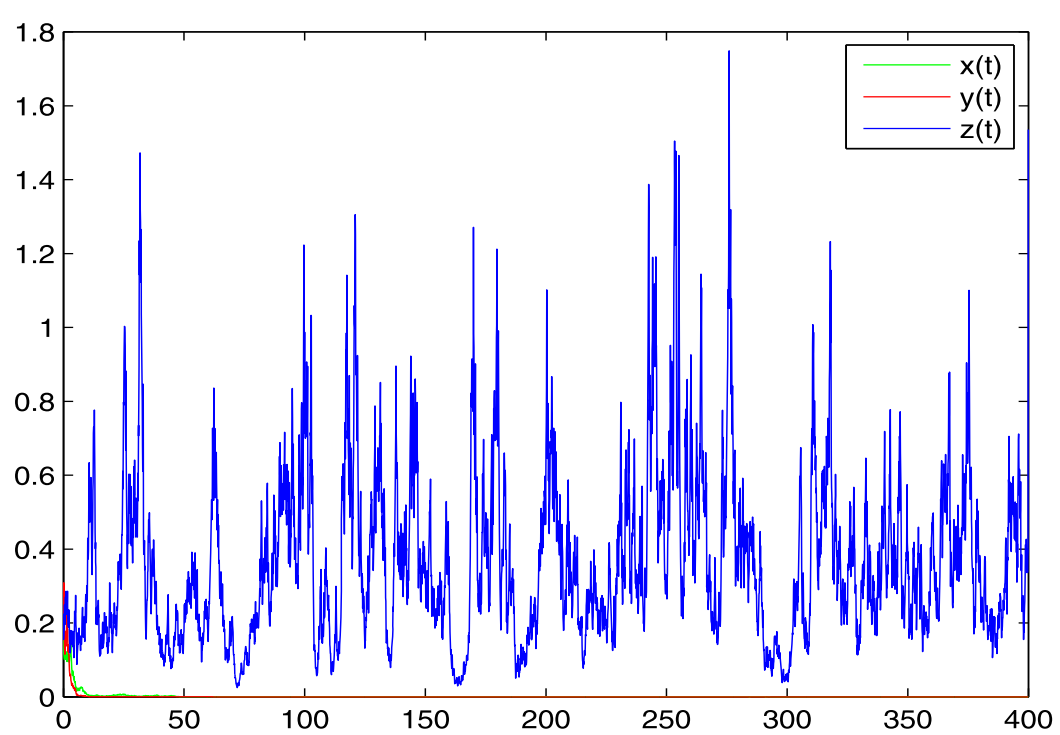

Figure 2 Solutions of system (3) for $a_{1}=0.02, a_{2}=0.03, a_{3}=0.5, \gamma_{1}(u)=\gamma_{2}(u)=0.2, \gamma_{3}(u)=0.4$, the other parameters remain the same as in (23), with step size $\Delta t=0.1>0$

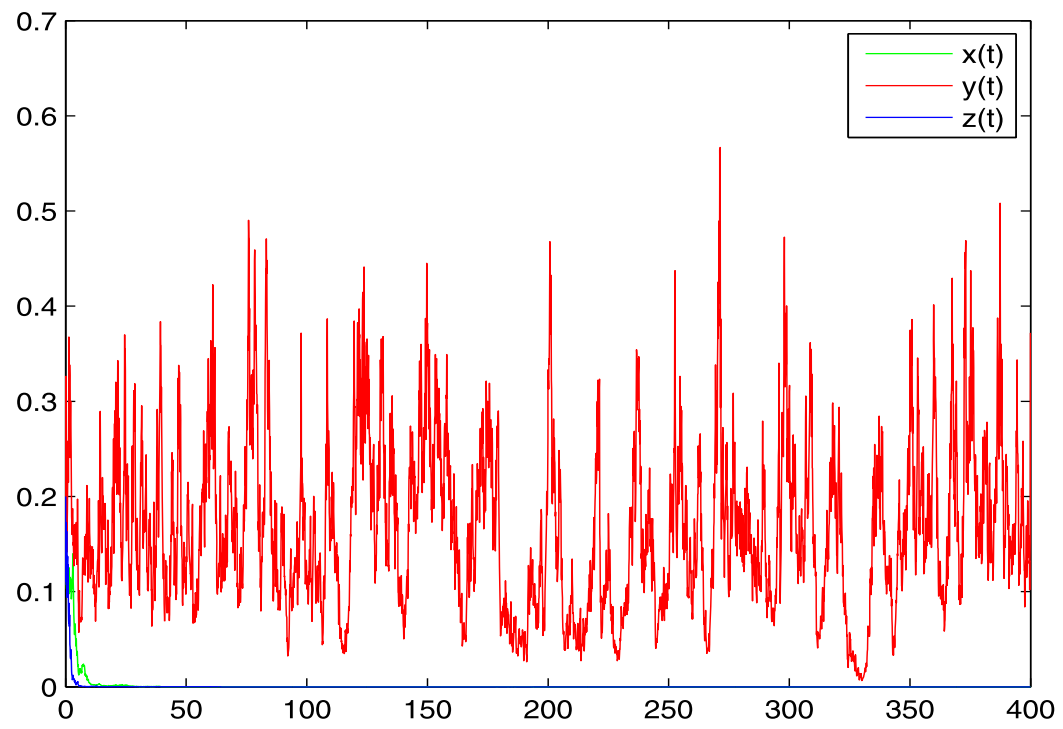

Figure 3 Solutions of system (3) for $a_{1}=0.06, a_{2}=0.6, a_{3}=0.2, \sigma_{3}=0.8, \beta=k_{2}=0.2, \gamma_{1}(u)=0.4$, $\gamma_{2}(u)=\gamma_{3}(u)=0.2$, the other parameters are the same as in (23), with step size $\Delta t=0.1>0$

By the condition of (iii)(a), the prey population $x(t)$ and the infected population $z(t)$ will go to extinction, the susceptible predator population $y(t)$ will be stable in the mean.

(4) In Figure 4, we choose $a_{1}=0.3, a_{2}=0.03, a_{3}=0.02, \gamma_{1}(u)=0.2, \gamma_{2}(u)=\gamma_{3}(u)=0.4$, the other parameters are the same as in (23), then

$$
w_{1}=0.1573>0, \quad w_{2}=-0.1585<0, \quad w_{3}=-0.1685<0 .
$$




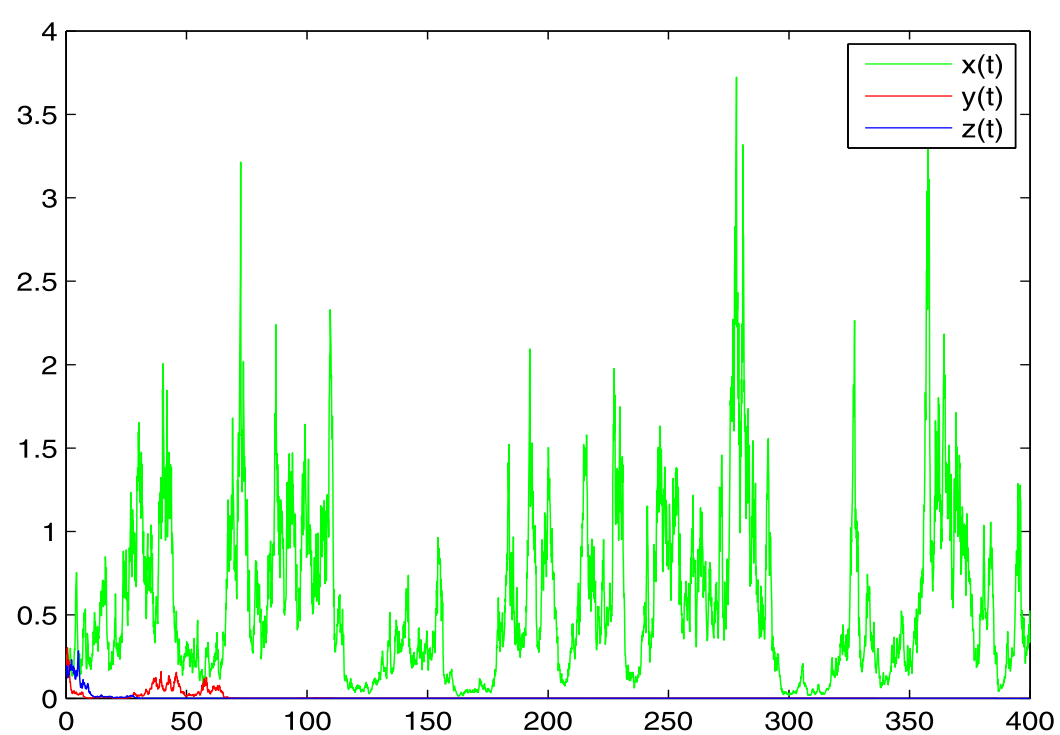

Figure 4 Solutions of system (3) for $a_{1}=0.3, a_{2}=0.03, a_{3}=0.02, \gamma_{1}(u)=0.2, \gamma_{2}(u)=\gamma_{3}(u)=0.4$, the other parameters are the same as in (23), with step size $\Delta t=0.1>0$

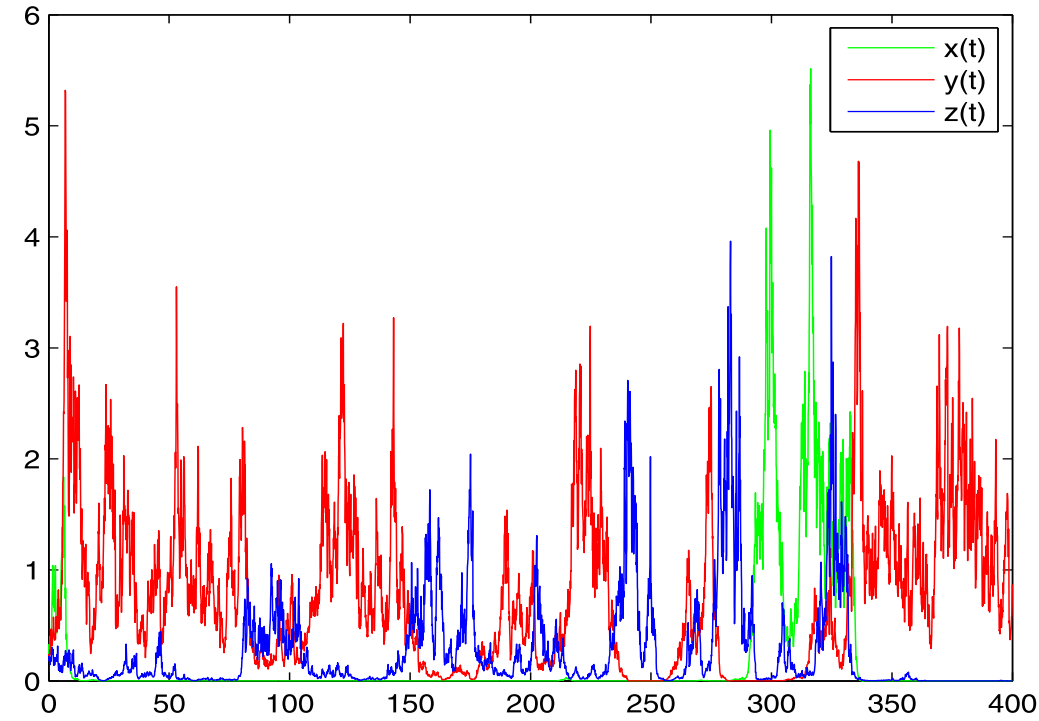

Figure 5 Solutions of system (3) for $a_{1}=0.75, a_{2}=0.7, a_{3}=0.3, c_{2}=0.3, c_{3}=0.2, k_{2}=0.6, \beta=0.4, \sigma_{3}=0.7$, $\gamma_{1}(u)=0.4, \gamma_{2}(u)=\gamma_{3}(u)=0.2$, the other parameters are the same as in (23), with step size $\Delta t=0.1>0$

On the basis of (iv) in Theorem 2, the prey population will be stable in the mean, the whole predator population will die out, which is consistent with Figure 4.

(5) In Figure 5, let $a_{1}=0.75, a_{2}=0.7, a_{3}=0.3, c_{2}=0.3, c_{3}=0.2, k_{2}=0.6, \beta=0.4$, $\sigma_{3}=0.7, \gamma_{1}(u)=0.4, \gamma_{2}(u)=\gamma_{3}(u)=0.2$, the other parameters are the same as in (23), then

$$
w_{1}=0.5615>0, \quad w_{2}=0.5573>0, \quad w_{3}=0.0373>0,
$$



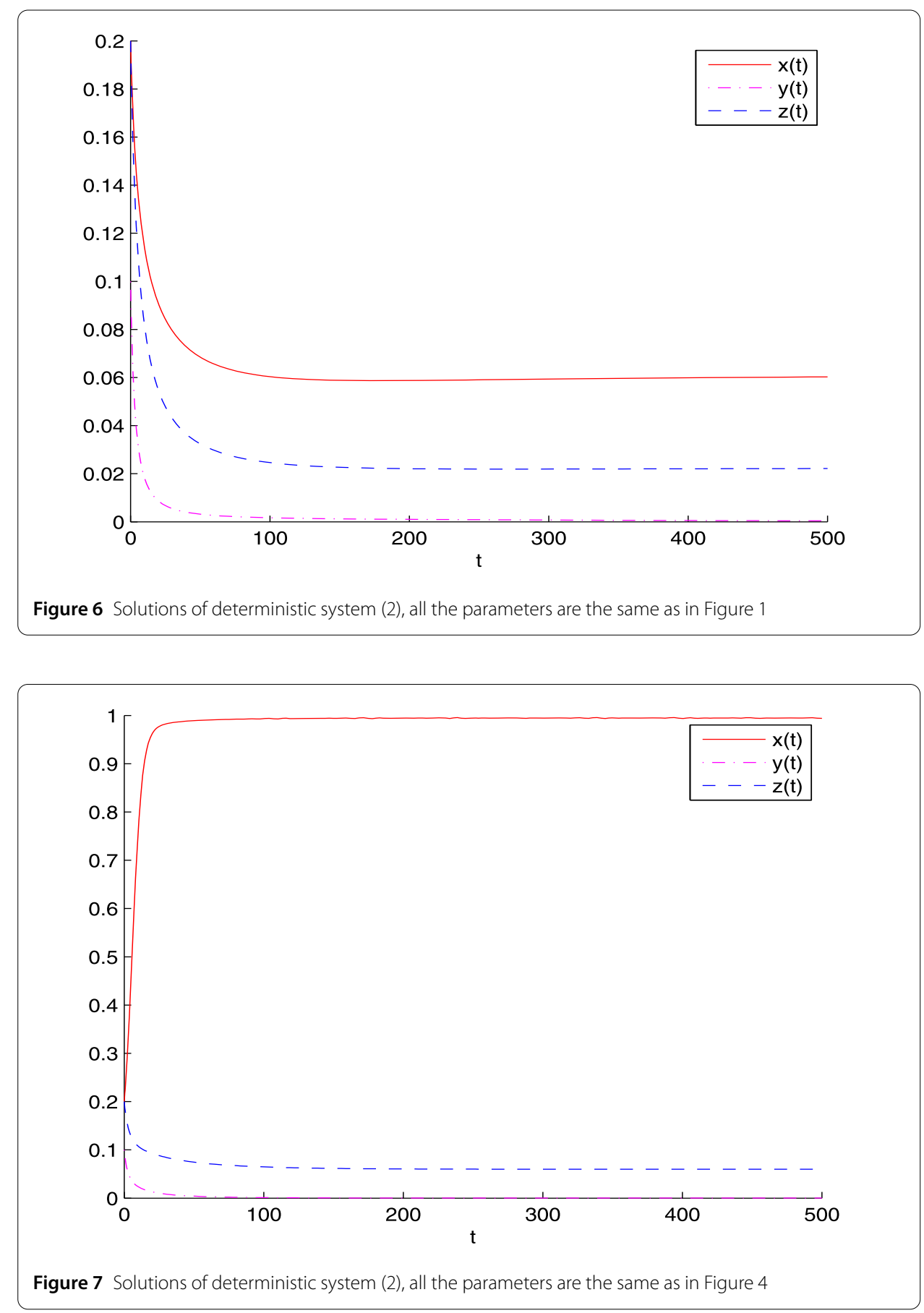

and $\frac{\left(w_{2}+w_{3}\right) k_{2} c_{1}}{\left(c_{2}+c_{3}+k_{2} \beta\right) k_{1}}=0.3857, w_{1}>\frac{\left(w_{2}+w_{3}\right) k_{2} c_{1}}{\left(c_{2}+c_{3}+k_{2} \beta\right) k_{1}}$. By virtue of $(\mathrm{v})$ in Theorem 2 , all the populations will be strongly persistent in the mean.

In the following, we discuss the solution of deterministic system (2), assume that all the parameters are the same as in Figures 1, 4, 5. Figure 6 shows that the susceptible predator population will be extinct, but the prey and infected predator populations will be stable, which is different from Figure 1.

Figure 7 illustrates that the prey population and the infected predator population will be stable, and the susceptible predator population will go to extinction. Compare Figure 7 


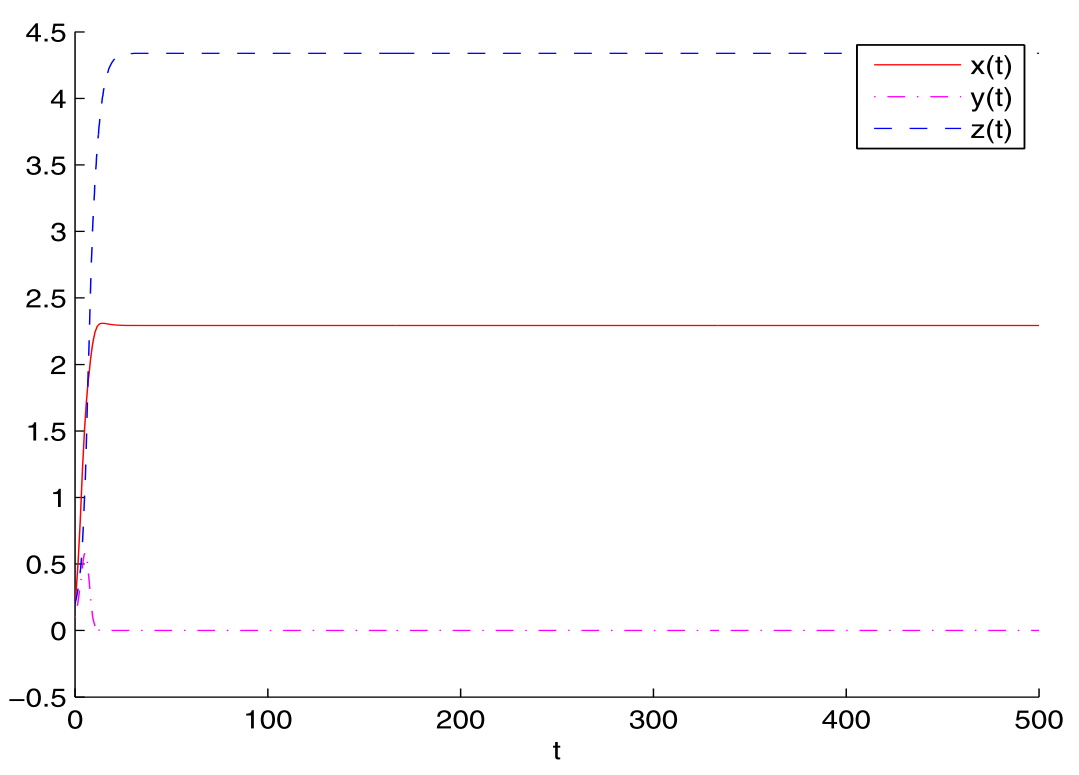

Figure 8 Solutions of deterministic system (2), all the parameters are the same as in Figure 5

with Figure 4, the behavior of solution for system (2) is distinct from that for stochastic system (3). Figure 8 confirms it again, we give no more explanations.

These results show that, for a stochastic system, not only the growth rate but also the Lévy noise and Gauss white noise play an important role in the persistence of population. Sometimes, the stochastic perturbation of environment can make species extinct.

\section{Conclusions}

In this paper, we discuss an LG-Holling type II diseased predator ecosystem with Lévy noise and Gauss white noise. We show that the system admits a unique global positive solution; we also investigate persistence in the mean and extinction of all the populations, uniformly finite $p$ th moment with $p>0$ and stochastic ultimate boundedness. The threshold conditions of extinction or persistence in the mean for prey and predator populations illustrate that $w_{i}$ is the key value, which means that $a_{i}$ is advantageous to the population, white noise and Lévy noise will go against the persistence of population. From the numerical simulation, we find that Lévy noise and Gauss white noise have an important effect on persistence for a stochastic system; occasionally, it may be a determinant factor. Thus, we must consider random changes of environment in the mathematical model.

Acknowledgements

This work is supported by the key research project of institutions of higher education of Henan Province in China (No. 18A110021)

Competing interests

The author declares that no competing interests exist.

Authors' contributions

The author has read and approved the final manuscript.

\section{Publisher's Note}

Springer Nature remains neutral with regard to jurisdictional claims in published maps and institutional affiliations. 
Received: 15 October 2017 Accepted: 18 January 2018 Published online: 02 February 2018

\section{References}

1. Leslie, P.H.: Some further notes on the use of matrices in population mathematics. Biometrika 35, 213-245 (1948)

2. Leslie, P.H.: A stochastic model for studying the properties of certain biological systems numerical methods. Biometrika 45, 16-31 (1958)

3. Pielou, E.C.: Mathematical Ecology. Wiley, New York (1977)

4. Korobeinikov, A.: A Lyapunov function for Leslie-Gower predator-prey models. Appl. Math. Lett. 14,697-699 (2001)

5. Zhou, X., Cui, J., Shi, X., Song, X.: A modified Leslie-Gower predator-prey model with prey infection. J. Appl. Math. Comput. 33, 471-487 (2010)

6. Liu, M., Wang, K.: Dynamics of a Leslie-Gower Holling-type II predator-prey system with Lévy jumps. Nonlinear Anal. $85,204-213(2013)$

7. Flores, J.D., Gonzalez-Olivares, E.: A modified Leslie-Gower predator-prey model with ratio-dependent functional response and alternative food for the predator. Math. Methods Appl. Sci. 40, 2313-2328 (2017)

8. Xu, Y., Liu, M., Yang, Y.: Analysis of a stochastic two-predators one-prey system with modified Leslie-Gower and Holling-type II schemes. J. Math. Anal. Appl. 7, 713-727 (2017)

9. Sarwardi, S., Haque, M., Venturino, E.: Global stability and persistence in LG-Holling type II diseased predator ecosystems. J. Biol. Phys. 37, 91-106 (2011)

10. Bao, J., Mao, X., Yin, G., Yuan, C.: Competitive Lotka-Volterra population dynamics with jumps. Nonlinear Anal. 74, 6601-6616 (2011)

11. Bao, J., Yuan, C.: Stochastic population dynamics driven by Lévy noise. J. Math. Anal. Appl. 391, 363-375 (2012)

12. Liu, M., Deng, M., Do, B.: Analysis of a stochastic logistic model with diffusion. Appl. Math. Comput. 266, 169-182 (2015)

13. Liu, Q., Chen, Q:: Asymptotic behavior of a stochastic non-autonomous predator-prey system with jumps. Appl. Math. Comput. 271, 418-428 (2015)

14. Liu, M., Wang, K.: Stochastic Lotka-Volterra systems with Levy noise. J. Math. Anal. Appl. 410, 750-763 (2014)

15. Zhao, Y., Yuan, S.: Optimal harvesting policy of a stochastic two-species competitive model with Levy noise in a polluted environment. Physica A 477, 20-33 (2017)

16. Liu, L., Meng, X., Zhang, T.: Optimal control strategy for an impulsive stochastic competition system with time delays and jumps. Physica A 477, 99-113 (2017)

17. Liu, Q., Jiang, D., Shi, N., Hayat, T., Alsaedi, A.: Stochastic mutualism model with Levy jumps. Commun. Nonlinear Sci. Numer. Simul. 43, 78-90 (2017)

18. Peng, S., Zhu, X.: Necessary and sufficient condition for comparison theorem of 1-dimensional stochastic differential equations. Stoch. Process. Appl. 116, 370-380 (2006)

19. Kunita, H.: Itố's stochastic calculus: its surprising power for applications. Stoch. Process. Appl. 120, 622-652 (2010)

20. Protter, P., Talay, D.: The Euler scheme for Lévy driven stochastic differential equations. Ann. Probab. 25, 393-423 (1997)

\section{Submit your manuscript to a SpringerOpen ${ }^{\circ}$ journal and benefit from:}

- Convenient online submission

- Rigorous peer review

- Open access: articles freely available online

- High visibility within the field

Retaining the copyright to your article

Submit your next manuscript at $\gg$ springeropen.com 